ARTIGO ORIGINAL

\title{
Análise dos efeitos de um programa de intervenção baseado na estrutura TARGET e "Escola da Bola" na coordenação motora grossa
}

\author{
Analyses of the effects of an intervention program based on TARGET structure and "Escola da Bola" \\ on the gross motor coordination
}

\author{
Maria Clara Soares de Oliveira Vaz ${ }^{1}$, Wagner de Campos ${ }^{2}$, Marcio José Kerkoski ${ }^{1}$, Gilmar Afonso ${ }^{1}$ \\ ${ }^{1}$ Universidade Tecnológica Federal do Paraná (UTFPR), Curitiba, Brasil \\ ${ }^{2}$ Universidade Federal do Paraná (UFPR), Curitiba, Brasil
}

\section{HISTÓRICO DO ARTIGO \\ Recebido: 30 setembro 2020 \\ $1^{\text {a }}$ Revisão: 28 novembro 2020 \\ $2^{a}$ Revisão: 25 janeiro 2021 \\ Aprovado: 17 fevereiro 2021 \\ PALAVRAS-CHAVE: \\ Criança; Intervenção Precoce; Desempenho Psicomotor.}

\section{KEYWORDS:}

Child; Early Intervention;

Psychomotor Performance.

\section{RESUMO}

OBJETIVO: O objetivo do trabalho foi analisar os efeitos de um novo programa de intervenção na coordenação motora grossa (CMG) de crianças entre os 05 e 10 anos, avaliadas pela bateria de testes KTK.

MÉTODOS: O programa foi realizado em 73 estudantes, selecionados de maneira não probabilística objetiva, com idades entre 05 e 10 anos, de uma rede municipal de ensino da cidade de Curitiba/PR, Brasil, divididos em dois grupos: Grupo Controle $(G C=35)$ e Grupo Intervenção $(G I=38)$. A CMG foi avaliada antes e depois da intervenção por meio da bateria de testes KTK. O programa de intervenção consistiu em 12 semanas de atividades alicerçadas nas estruturas da Técnica de Motivação Orientada Para Maestria (TARGET), e no livro "Escola da Bola - Um ABC para Iniciantes nos Jogos Esportivos". Os efeitos foram testados utilizando o Teste de Cohen e teste $t$ (dependente e independente).

RESULTADOS: Na testagem $t$ dependente, diferenças estatisticamente significativas foram encontradas no GI, do primeiro para o segundo momento, em todos os testes ( $d \geq 0,80)$. O Gl obteve uma melhora significativa ao longo das 12 semanas $(p<0,05)$, enquanto no GC não houve diferenças estatisticamente significativas. Na testagem $t$ independente, no momento pré-intervenção (1 Momento), foram encontradas diferenças significativas $(p<0,05)$ nas quatro tarefas, favoravelmente ao GC. No momento pós-intervenção, diferenças estatisticamente expressivas não foram observadas entre o Gl e GC.

CONCLUSÃO: Conclui-se que um programa interventivo, com duração de 12 semanas, utilizando os jogos da "Escola da Bola" em conjunto com o "TARGET", trazem melhorias significativas no desempenho coordenativo das crianças, entre 5 e 10 anos de idade.

\section{ABSTRACT}

OBJECTIVE: The objectives of the work were to analyze the effects of a new intervention program on gross motor coordination (GMC) of 5 and 10 year old children, diagnosed by the KTK battery test.

METHODS: The program was carried out in 73 students, selected in an objective non-probabilistic way, aged between 5 and 10 years, from a municipal education network in the city of Curitiba, Brazil, divided into two groups: Control Group ( $C G=35)$ and Intervention Group ( $I G=38)$. CMG was assessed before and after the intervention using the KTK test battery. The intervention program consisted in 12 weeks of activities based on the structures of the Mastery-Oriented Motivation Technique (TARGET), and the book "Escola da Bola - Um $A B C$ para Iniciantes nos Jogos Esportivos". The effects were tested using the Cohen test and $t$-test (dependent and independent).

RESULTS: In the dependent $t$-test, statistically significant differences were found on the IG, from the first to the second moment, in all tests $(d \geq 0.80)$. The IG achieved a significant improvement over the 12 weeks $(p<0,05)$, while in the CG there were no statistically significant differences. In the independent $t$-test, in the pre-intervention moment (1st Moment), significant differences $(p<0,05)$ were found on the four tasks, in favor of the CG. In the post-intervention moment, statistically significant differences were not observed between the IG and CG.

CONCLUSION: It follows that an intervention program, lasting 12 weeks, using the games of "Escola da Bola" together with "TARGET", brings significant improvements in children's coordinative performance, between 5 and 10 years old. 


\section{INTRODUÇÃo}

De fato, a palavra "criança" sempre esteve relacionada ao movimento. Entretanto, mudanças severas no quotidiano das famílias - causadas pela violência urbana, o crescente uso de tecnologias, a falta de tempo livre e a limitação de espaços de lazer - têm alterado o estilo de vida destas, afetando negativamente a forma com que se movimentam (FERRARI, 2013; ARAÚJO, 2000).

Neste contexto, níveis insuficientes de atividade física são relatados a nível global em todas as idades, incluindo a primeira infância (HALLAL, 2012; LEE, 2012). No Brasil, um estudo conduzido por Reis e colaboradores (2009), analisou a associação entre o nível de atividade física e a percepção dos jovens em relação aos espaços públicos. A amostra, composta por 671 rapazes e 979 moças, mostrou que, preocupantemente, 78,3\% e $90,9 \%$ destes não atingiam o tempo mínimo (sessenta minutos diários) de atividade física recomendada, respectivamente. Em consequência do vigente cenário, a coordenação motora grossa (CMG) dos indivíduos, influenciada por diversas determinantes (biológicas, físicas, da tarefa e sociais), descende preocupantemente (NEWELL, 1989; LOPES et al., 2003). Estudos mostram que crianças com melhor desempenho coordenativo estão mais dispostas a adotarem um estilo de vida ativo, dando continuidade às práticas esportivas ao longo de sua vida (STODDEN et al., 2008; LAI, 2014).

O sujeito, ao adotar um estilo de vida ativo, diminui significativamente, a curto e a longo prazo, a probabilidade de desenvolver doenças cardiovasculares e ósseas, obesidade, diabetes e pressão alta (LEE, 2012; BASSUK; MANSON, 2005; PIOLA et al., 2018). Além disso, pesquisas mostram que atrasos no desenvolvimento motor estão relacionados a dificuldades na aprendizagem escolar. Bart, Hajami e BarHaim (2007), ao analisarem o desempenho motor de 88 crianças, verificaram que, bons níveis de habilidades motoras, facilitam o ajuste dos indivíduos ao ambiente escolar, proporcionando a estes um melhor convívio social e obtenção de notas satisfatórias. Dado o fato que o ambiente escolar engloba a criança em seu período sensível, este configura-se como um excelente espaço para a realização de intervenções que busquem melhorar a CMG das crianças, trazendo benefícios também para áreas adjacentes à atividade escolar.

Tendo em vista os benefícios causados pela prática de atividades físicas - bem como os malefícios provenientes do baixo desempenho coordenativo - programas de intervenção que objetivam melhorias nos níveis de CMG têm sido alvo de estudo de diversos pesquisadores, sendo de extrema importância no período da infância (MALINA; BOUCHARD; BAR-OR, 2009). Em uma revisão sistemática, Dobbins et al. (2013), analisaram cinco estudos que avaliaram o impacto das intervenções motoras, aplicadas no ambiente escolar, sobre o nível de atividade física dos sujeitos. Entre os resultados reportados, observou-se que obtiveram efeitos satisfatórios as intervenções administradas por um período de, no mínimo, 12 semanas, com duração entre 20 a 50 minutos por sessão (IIVONEN; SAAKSLAHTI, 2014).

A fim de obter-se melhorias significativas no desempenho motor dos indivíduos, ao longo dos anos, diversos autores vêm investigando diferentes métodos interventivos para tal população. A "Descoberta Dirigida" (MOSSTON; ASHWORTH, 1986), amplamente utilizada na década de 80 e 90 por profissionais da educação física (BAÑUELOS, 1989; CONTRERAS-JÓRDAN, 1998), consiste na imposição de desafios ao indivíduo, oferecendo-o a possibilidade de solucioná-los por conta própria - promovendo assim a aprendizagem e retenção de novos padrões motores. Apesar dos resultados satisfatórios, pesquisas que utilizaram a "Descoberta Dirigida" como a principal abordagem interventiva são muito recentes, necessitando-se mais estudos para comprovação de sua replicabilidade (MORERA-CASTRO; ARGUEDAS-VIQUEZ; BRABENEC-AGUILAR, 2020).

Ainda na década de 80, a Abordagem Desenvolvimentista é trazida ao Brasil, apresentando novas maneiras de se intervir no movimento dos sujeitos (TANI et al., 1988). Neste contexto, intervenções que utilizavam técnicas combinadas, ou seja, não eram pautadas em estratégias pré-definidas, e que tinham como foco principal o movimento, ganharam popularidade (BRAGA et al., 2009; POETA; ROSA NETO, 2005; CAMPOS et al., 2008). Posteriormente, outros métodos interventivos passaram a ser difundidos, os quais podemos citar: intervenções baseadas em ações motoras (futebol, vôlei, dança, natação etc.), intervenções baseadas nas habilidades motoras (jogar, pegar, pular etc.) e, por fim, as intervenções baseadas em videogames (SOUZA; BERLEZE; VALENTINI, 2008; FERNANDES; MOURA; SILVA, 2017; CARO et al., 2017; LARKIN; HOARE; SMITH, 1989).

Quanto as metodologias aplicadas em programas interventivos direcionados à indivíduos com desordem coordenativa, estudos verificaram que há três abordagens extensamente utilizadas, sendo estas: Abordagem Orientada Para o Processo (treinamento cinestésico e integração sensorial); Abordagem Orientada Para a Tarefa (focadas no desempenho e aprendizagem motora) e Terapia Física e Convencional Tradicional (SMITSENGELSMAN et al., 2013; PLESS; CARLSSON, 2000).

Neste sentido, uma estratégia interventiva que trabalha os interesses do sujeito, suas habilidades motoras e é orientada para tarefa; passou a ser reconhecida por estudiosos em todo mundo - principalmente no Brasil. A estrutura "TARGET", criada por Ames (1987), é descrita como uma abordagem educacional, centrada na autonomia da criança e no desejo intrínseco de tornar-se competente na tarefa proposta (AMES, 1992). A sigla "TARGET" faz alusão, em cada letra, a uma parte elementar da estrutura, sendo estas: Tarefa, Autoridade, Reconhecimento, Grupo, Avaliação e Tempo (VALENTINI, 2002). Tal estratégia, ao ser aplicada em crianças, obteve resultados satisfatórios, no que diz respeitos à CMG e habilidades motoras fundamentais (GOODWAY; HAUBENSTRUCKER, 1999; LOPES; MAIA, 1997; COSTA, 2014; VALENTINI; RUDISILL, 2006; PÍFFERO; VALENTINI, 2010; LAGE, 2011).

Entretanto, como dito anteriormente, desde o final da década de 80 , no Brasil, busca-se inovar os programas interventivos. Sendo assim, a aplicação da metodologia "TARGET", individualmente, não se configura como uma estratégia interessante e inovadora. Logo, outra metodologia, com foco nas ações motoras, foi selecionada para montagem do presente estudo. A "Escola da Bola - um ABC para Iniciantes nos Jogos Esportivos", tem por objetivo permitir que as crianças experienciem, de forma rica e variada, diferentes alternativas de movimento, de forma universal, apresentando em sua estrutura três pilares fundamentais; que dispõe de objetivos, conteúdos e metas específicas a serem alcançadas durante o processo de ensino dos jogos (ROTH; KROGER, 2006). Ao ser aplicada no cenário brasileiro, a "Escola da Bola" obteve resultados satisfatórios relacionados à 
aprendizagem e melhoria da coordenação motora grossa e das habilidades motoras fundamentais (ABURACHID et al., 2015; CABRAL; ABURACHID; GRECO, 2012; MEMMERT; ROTH, 2006; ABURACHID et al., 2019). Assim sendo, acredita-se que a aprendizagem por meio de jogos e brincadeiras (ROTH; KROGER, 2006), em conjunto com a estratégia "TARGET" (AMES, 1987), demonstra as qualificações metodológicas necessárias para a estruturação de um programa de desenvolvimento coordenativo eficaz - a ser aplicado em um ambiente escolar.

Considerando-se o crescente número de crianças com atrasos no desempenho coordenativo, emerge a necessidade de novos e eficazes métodos interventivos, a fim de mudar-se tal cenário. Visto que, no Brasil, não há registro de estudos que tenham utilizado a estratégia "TARGET", em conjunto com a "Escola da Bola", como meio interventivo; o objetivo do presente estudo foi analisar os efeitos de um novo programa de intervenção na coordenação motora grossa (CMG) de crianças entre os 5 e 10 anos de idade, avaliadas pela bateria de testes KTK.

\section{MÉTODOS}

Os participantes do presente estudo foram estudantes disponibilizados por uma Unidade de Ensino Integral (UEI) da rede municipal de ensino da cidade de Curitiba (PR), Brasil, com idade entre os 5 e 10 anos. Foram excluídos do estudo alunos com deficiência física, visual, auditiva ou intelectual; com frequência menor que $70 \%$ nas aulas e que não apresentaram o Termo de Consentimento Livre e Esclarecido assinado pelos pais ou responsáveis.

Após exclusão, 73 participantes, selecionados de maneira não probabilística, objetiva (MAROCO, 2003), foram avaliados por meio da bateria motora alemã Körperkoordinationstest Für Kinder - KTK (KIPHARD; SCHILLING, 1974), administrada em dois momentos distintos, sendo um antes da intervenção (pré-teste, primeiro momento) e outro ao final desta (pós-teste, segundo momento). Após a primeira avaliação, verificou-se, por meio dos valores discretos das médias em cada tarefa, que as crianças do turno vespertino apresentavam um desempenho individual inferior nas tarefas do KTK - sendo designadas, portanto, ao Grupo de Intervenção (GI), consequentemente, agrupando os indivíduos do turno matutino no Grupo Controle (GC). O GI e o GC foram compostos, respectivamente, por 38 (21 meninas e 17 meninos) e 35 (14 meninas e 21 meninos) indivíduos. Garantiu-se o anonimato e confidencialidade dos dados dos participantes.

O KTK pode ser aplicado em crianças com idade entre cinco e quatorze anos, tendo duração de execução de aproximada-mente quinze minutos por avaliado. A avaliação constitui-se por meio de quatro tarefas, sendo estas: Trave de Equilíbrio, Saltos Monopedais, Saltos Laterais e Transferência sobre Plataforma (KIPHARD; SCHILLING, 1974). Ao final, sugere-se transformar os valores brutos obtidos em um quociente motor, classificando a coordenação motora da criança em cinco níveis distintos.

Entretanto, apesar do quociente motor ter sido utilizado como parâmetro em alguns estudos (MOREIRA et al., 2019), pelo fato de haver controvérsias acerca da validade transcultural dos valores estandardizados pelos autores alemães, no presente estudo, o quociente motor não foi calculado, conside- rando-se, portanto, o desempenho do indivíduo em cada tarefa (DEUS et al., 2010; VIDAL et al., 2009).

Referente à estrutura metodológica adotada para intervenção, na presente pesquisa, propõe-se um programa que considere as capacidades e interesses das crianças - obtendo melhorias significativas no desempenho coordenativo destas. Selecionaram-se então os Jogos Orientados Para Situação (Pilar A da Escola da Bola), que intentam ensinar o indivíduo a jogar de maneira global, objetivando a redução dos déficits coordenativos. Neste pilar, os jogos são realizados com certo grau de liberdade, fazendo os sujeitos adquirirem capacidades gerais de jogo e determinada competência tática (ROTH; KROGER, 2006). Para prática de tais atividades dentro do programa de intervenção, montou-se uma "caixa de jogos", posteriormente incorporada à estrutura "TARGET", no componente "Task" (tarefa). Logo, os jogos propostos no pilar A da "Escola da Bola" foram selecionados de acordo com as valências motoras a serem treinadas ("caixa de jogos"), e distribuídos de forma que puderam ser executados com diversas variações, dentro do quesito das Tarefas da estrutura "TARGET", de modo a trabalhar com eficiência a coordenação motora grossa, desenvolvendo-a.

Semelhantemente a estrutura "TARGET", os jogos da "Escola da Bola" são realizados em equipe. Posto isto, os "Grupos" foram divididos pelos próprios alunos, porém com a exigência da formação de grupos heterogêneos, entre meninos e meninas. Formados os grupos, os indivíduos auxiliaram a professora com sugestões para variações e regras a serem aplicadas durante a execução dos jogos ("Tarefa") - efetivando assim sua "Autoridade". No decorrer das atividades, reforços positivos eram concedidos de forma constante às crianças participantes ("Reconhecimento"), respeitando-se sempre o "Tempo" individual de cada um, independente do caráter coletivo do jogo; visto que as variações da tarefa só avançaram quando todos da turma se sentiram preparados para tal. Por fim, a "Avaliação" dos alunos ocorreu ao final de cada aula, por meio de registros acerca das evoluções e conquistas adquiridas pelos sujeitos, bem como sugestões para as aulas conseguintes.

Deste modo, o programa interventivo foi aplicado em um ambiente escolar, no período extracurricular, por meio de 12 aulas semanais de jogos com caráter coletivo, com 50 min de duração por sessão, contendo variações na execução do movimento e no nível de complexidade de execução. Ao final, realizou-se uma comparação entre os resultados pré e pós-intervenção, obtidos por meio da pontuação das tarefas da bateria de testes KTK, a fim de se averiguar a eficácia do programa.

Para análise dos dados dos participantes, após a confirmação da normalidade da distribuição de dados, por meio do Teste de Kolmogorov-Smirnov, testes paramétricos foram utilizados. Medidas descritivas (média e desvio-padrão) foram calculadas. A avaliação do impacto do projeto de intervenção sob os níveis de coordenação motora grossa foi realizada por meio do "Teste de Cohen" e do "Teste T", comparando-se os resultados obtidos na bateria motora do KTK, no primeiro e no segundo momento de intervenção, entre o grupo intervenção e controle (independente) e entre os próprios grupos (dependente). A análise dos dados ocorreu por meio do programa Statistical Package for the Social Sciences (SPSS), versão 23.0, com valor de significância $\mathrm{p}<0,05$ e $95 \%$ I.C. 


\section{RESULTADOS E DISCUSSÃO}

Ao analisar a significância dos resultados das médias das quatro tarefas que compõem o KTK, nomeadamente, Salto Lateral, Salto Monopedal, Equilíbrio à Retaguarda e Transposição Lateral, no primeiro e segundo momento de avaliação, observa-se no Grupo de Intervenção (GI), um incremento maior que no Grupo Controle (GC) (Tabela 1). A significância dos resultados foi calculada por meio do "Teste de Cohen", onde o tamanho do efeito (d) foi classificado da seguinte forma: 0,20-0,30 (pequeno), 0,40-0,70 (médio), \0,80 (grande) (BISQUERRA; SARRIERA; MARTINEZ, 2004).

Tabela 1. Medidas inferenciais e teste t dependente no primeiro e segundo momento de avaliação.

\begin{tabular}{|c|c|c|c|c|}
\hline \multirow[b]{3}{*}{ Grupos } & \multicolumn{2}{|c|}{ Momentos } & \multirow[b]{3}{*}{$\mathrm{p}$} & \multirow[b]{3}{*}{$d^{*}$} \\
\hline & $1^{\circ}$ Momento & $2^{\circ}$ Momento & & \\
\hline & Média $\pm D P$ & Média $\pm D P$ & & \\
\hline \multicolumn{5}{|l|}{ Intervenção - GI (tarefas) } \\
\hline Transposição Lateral & $23 \pm 8,85$ & $30,17 \pm 7,77$ & 0,000 & 6,29 \\
\hline Equilíbrio à Retaguarda & $21,58 \pm 12,5$ & $30,26 \pm 14,68$ & 0,000 & 10,19 \\
\hline Salto Monopedal & $26,18 \pm 14,46$ & $31,46 \pm 11,85$ & 0,003 & 4,32 \\
\hline Salto Lateral & $37,16 \pm 17,26$ & $44,83 \pm 15,82$ & 0,000 & 7,03 \\
\hline \multicolumn{5}{|l|}{ Controle - GC (tarefas) } \\
\hline Transposição Lateral & $33,23 \pm 11,55$ & $34,27 \pm 10,58$ & 0,029 & 0,95 \\
\hline Equilíbrio à Retaguarda & $29,83 \pm 18,88$ & $28,46 \pm 12,27$ & 0,717 & $-0,89$ \\
\hline Salto Monopedal & $38,74 \pm 16,86$ & $37,42 \pm 14,56$ & 0,313 & $-1,13$ \\
\hline Salto Lateral & $44,89 \pm 16,84$ & $45,03 \pm 22,92$ & 0,650 & 0,19 \\
\hline
\end{tabular}

Observa-se valores com alto grau de significância para o Gl $(d \geq 0,80)$ em todas as tarefas; em contraponto, no GC, apesar do aumento discreto nos valores médios, tal variação não foi estatisticamente relevante no Equilíbrio à Retaguarda $(\mathrm{d}=-0,89)$, Salto Monopedal $(d=-1,13)$ e Salto Lateral $(d=0,19)$.

Semelhantemente, ao realizar-se o "Teste T Dependente" (Tabela 1), expresso pelo " $p$-value", diferenças estatisticamente significativas são percebidas no $\mathrm{Gl}$, em todas as tarefas $(p<0,05)$. No GC, diferenças estatisticamente relevantes foram encontradas apenas na Transposição Lateral $(p=0,029)$. Tal discrepância entre os grupos pode ocorrer em consequência dos estímulos e atividades proporcionados pelo programa de intervenção, visto que o Gl obteve mais experiências motoras ao longo das 12 semanas, tendo a possibilidade de aprimorar sua CMG, enquanto o GC manteve a rotina de atividades físicas entre o primeiro e segundo momento de avaliação.

Zanella e colaboradores (2016), ao analisarem o impacto de uma intervenção motora nos níveis de CMG de crianças com sobrepeso e obesidade, evidenciaram queda no desempenho coordenativo dos indivíduos pertencentes ao GC. Esta variação pode ser explicada pela baixa estabilidade da variável CMG (apresentada em estudos longitudinais prévios), e pela falta de estímulos motores propostos nas aulas de educação física, ressaltando-se assim a importância de um planejamento metodológico adequado (BASSO et al., 2012; LOPES, 1997). Considerando que tal variação não foi encontrada nos dois grupos, supõe-se que o programa de intervenção estimulou as crianças do Gl adequadamente, fornecendo a estas experiências motoras diversificadas, que foram utilizadas nas tarefas solicitadas. Pesquisas que aplicaram a "Escola da Bola" como meio interventivo, obtiveram resultados positivos semelhantes aos expostos no presente estudo (ABURACHID et al., 2019; ABURACHID et al., 2015; CABRAL; ABURACHID; GRECO, 2012; MEMMERT; ROTH, 2006). Analogamente, diferenças entre os resultados do grupo controle e do grupo intervenção, em estudos baseados na estrutura "TARGET", são amplamente relatadas (SAMPAIO; VALENTINI, 2015; LAZZARONI, 2017; ZANELLA et al., 2016; BERLEZE; HAEFFNER; VALENTINI, 2008).

Por meio da realização do "Teste T Independente" (Tabela 2), as diferenças entre os grupos, no primeiro e segundo momento de avaliação, foram verificadas. Observa-se que, no momento pré-intervenção ( $1^{\circ}$ momento), diferenças significativas foram encontradas entre os grupos, em todos os componentes do KTK $(p<0,05)$, favoravelmente aos maiores valores do GC. As diferenças foram evidenciadas pelo desempenho coordenativo inferior que o Gl apresentava, em relação ao GC, expresso pelos valores médios obtidos em cada tarefa. Entretanto, no segundo momento, diferenças estatisticamente relevantes não foram observadas.

Tabela 2. Teste $T$ Independente no primeiro e no segundo momento, no Grupo Intervenção (GI) e Grupo Controle (GC).

\begin{tabular}{|c|c|c|c|}
\hline \multirow[b]{3}{*}{ Momentos } & \multicolumn{2}{|c|}{ Grupos } & \multirow[b]{3}{*}{$\mathrm{p}$} \\
\hline & Intervenção (GI) & Controle (GC) & \\
\hline & Média $\pm D P$ & Média $\pm D P$ & \\
\hline \multicolumn{4}{|l|}{$1^{\circ}$ Momento (tarefas) } \\
\hline Transposição Lateral & $23,00 \pm 8,85$ & $33,23 \pm 11,55$ & 0,000 \\
\hline Equilíbrio à Retaguarda & $21,58 \pm 12,5$ & $29,83 \pm 18,88$ & 0,030 \\
\hline Salto Monopedal & $26,18 \pm 14,46$ & $38,74 \pm 16,86$ & 0,001 \\
\hline Salto Lateral & $37,16 \pm 17,26$ & $44,89 \pm 16,84$ & 0,057 \\
\hline \multicolumn{4}{|l|}{$2^{\circ}$ Momento (tarefas) } \\
\hline Transposição Lateral & $30,17 \pm 7,77$ & $34,27 \pm 10,58$ & 0,086 \\
\hline Equilibrio à Retaguarda & $30,26 \pm 14,68$ & $28,46 \pm 12,27$ & 0,605 \\
\hline Salto Monopedal & $31,46 \pm 11,85$ & $37,42 \pm 14,56$ & 0,094 \\
\hline Salto Lateral & $44,83 \pm 15,82$ & $45,03 \pm 22,92$ & 0,967 \\
\hline
\end{tabular}

Mediante tais dados, constata-se que, ao longo das 12 semanas interventivas, o GI melhorou seu desempenho coordenativo, equiparando-se ao GC. Os resultados encontrados são expectáveis, tendo em vista que pesquisas prévias comprovam que, ao longo do tempo, as crianças com os menores desempenhos iniciais são as que sofrem as maiores mudanças (BASSO et al., 2012). Com o objetivo de investigar se um programa de intervenção, orientado para maestria em Ginástica Rítmica (GR), influenciaria o desempenho coordenativo dos indivíduos; Sampaio e Valentini (2015), avaliaram 39 meninas, dos 5 aos 10 anos, separando-as em GI e GC. Concluíram que, após três meses do programa, embora o GI tenha apresentado melhoras expressivas, diferenças estatisticamente significantes não foram encontradas entre os grupos, corroborando com o resultado encontrado no presente estudo. Da mesma forma, Souza e colaboradores (2008), ao mensurarem o desempenho coordenativo de 26 crianças, após um programa baseado na estrutura "TARGET", não constataram diferenças expressivas entre o Gl e o GC. 
Logo, os dados do presente estudo evidenciam que, um programa de intervenção de 12 semanas, baseado na estrutura "TARGET" e na "Escola da Bola", é eficaz na melhora da CMG de crianças entre os 05 e 10 anos, no que diz respeito ao desempenho individual nas quatro tarefas que compõem o KTK. Isto se deve ao fato de que, os sujeitos expostos ao programa, vivenciaram uma grande variedade de ações motoras durante os jogos, de forma a aprimorar seu desempenho coordenativo. Ao se combinar os jogos da "Escola da Bola" com a estrutura "TARGET", os indivíduos envolveram-se semanalmente em jogos lúdicos cooperativos e competitivos, que visavam a superação de suas próprias limitações, a autonomia e o trabalho em grupo; resultando assim na melhora coordenativa.

Sugere-se, para estudos futuros, além da aplicação do projeto em amostras maiores, a análise de outras variáveis, tais como aspectos psicológicos, nível de aptidão física e aquisição de habilidades motoras, possibilitando uma pesquisa mais extensa em relação aos benefícios provenientes de tal combinação de estratégias motoras.

\section{CONCLUSÃO}

O Programa Interventivo, baseado em estratégias motivacionais ("TARGET") e jogos (Escola da Bola), promoveu a vivência de diversas ações motoras, o aprimoramento da coordenação motora grossa e a inclusão de todos os alunos na prática. Relativo ao desempenho coordenativo das crianças, evidenciou-se que a combinação das duas técnicas promove ganhos significativos na coordenação motora grossa. Tais resultados demonstram que jogos cooperativos e competitivos, quando orientados para maestria, por um período de 12 semanas, configuram-se como estratégias interessantes a serem implementadas pelo profissional de Educação Física, no ambiente escolar.

\section{REFERÊNCIAS}

ABURACHID, L. M. C.; SILVA, S. R.; ARAÚJO, N. D.; GRECO, J. P. Badminton: possibilidades de ensino aplicadas ao contexto da educação física escolar. Journal of Physical Education, Maringá, v. 30, n. 1, p. e-3055, 2019.

AMES, C. Classroom: goals, structures, and student motivation. Journal of Educational Psychology, Washington, v. 84, p. 261-71, 1992.

AMES, C. Enhancement of student motivation. In: KLEIBER, D. (Ed.) Advances in motivation and achievement. Jai Press, Greenwith, v. 5, n. 1, p. 123 48,1987

ARAÚJO, D. S. Aptidão física, saúde e qualidade de vida relacionada à saúde em adultos. Revista Brasileira de Medicina e Esporte, Niterói, v. 6, n. 5, p. 194-206, 2000

BART, O.; HAJAMI, D.; BAR-HAIM., Y. Predicting school adjustment from motor abilities in kindergarten. Infant And Child Development, Nova Jersey, v. 16 , n. 6, p. 597-615, 2007.

BASSO, L.; SOUZA, C. J. F.; ARAÚJO, U. O.; BASTOS, H. F.; BIANCHI, T. T.; MEIRA JÚNIOR, C. M.; OLIVEIRA, J. A.; PRISTA, A.; TANI, G.; MAIA, J. A. R. Olhares distintos sobre a noção de estabilidade e mudança no desempenho da coordenação motora grossa. Revista Brasileira de Educação Física e Esporte, São Paulo, v. 26, n. 3, p. 495-509, 2012.

BASSUK, S.; MANSON, E. Epidemiological evidence for the role of physical activity in reducing risk of type 2 diabetes and cardiovascular disease. Journal of Applied Physiology, Bethesda, v. 99, p. 1193-204, 2005.

BERLEZE, A.; HAEFFNER, L. S.; VALENTINI, N. C. Prevalência de obesidade na infância em diferentes agrupamentos sociais e a importância de estratégias pedagógicas. Saúde, Santa Maria, v. 34a, p. 44-9, 2008.

BISQUERRA, R.; SARRIERA, J.; MARTINEZ, F. Introdução à estatística: enfoque informático com o pacote estatístico SPSS. Porto Alegre: Artes Médicas: 2004.
BRAGA, R. K.; KREBS, R. J.; VALENTINI, N. C.; TKAC, C. M. A Influência de um programa de intervenção motora no desempenho das habilidades locomotoras de crianças com idade entre 6 e 7 Anos. Revista da Educação Física, Maringá, v. 20, , n. 2, p. 171-81, 2009

CABRAL, F. A.; ABURACHID, L. M. C.; GRECO, P. J. Futsal feminino universitário: análise de uma proposta metodológica para desenvolvimento das habilidades técnicas. Revista Mineira de Educação Física, Viçosa, v. 2, Edição Especial, n. 1, p. 1451-6, 2012

CARO, K.; TENTORI, M.; MARTINEZ-GARCIA, A. I.; ZAVALA-IBARRA, I. FroggyBobby: An exergame to support children with motor problems practicing motor coordination exercises during therapeutic interventions. Computers in Human Behavior, Amsterdam, v. 71, p. 479-98, 2017.

CONTRETAS-JÓRDAN, O. R. Ensino de educação física. Uma abordagem construtivista. Barcelona: Publicações INDE, 1998

COSTA, C. L. Efeito de um programa de intervenção motora sobre o desenvolvimento motor de crianças em situação de risco social na região do Cariri - CE. Revista da Educação Física, Maringá, v. 25, n. 3, p. 353-64, 2014.

DEUS, R. K.; BUSTAMANTE, A.; LOPES V. P.; SEABRA, A. T.; SILVA, R. M. G.; MAIA, J. A. R. Modelação longitudinal dos níveis de coordenação motora de crianças dos seis aos 10 anos de idade da Região Autônoma dos Açores, Portugal. Revista Brasileira de Educação Física e Esporte, São Paulo, v. 24 n. 2, p. 259-73, 2010.

DOBBINS, M.; HUSSON, H.; DECORBY, K.; LAROCCA, R. L. School-based physical activity programs for promoting physical activity and fitness in children and adolescents aged 6 to 18 . Cochrane Database of Systematic Reviews, Hoboken, v. 2, n. 2, p. 1-25, 2013.

FERNANDES, S. P.; MOURA, S. S.; SILVA, S. A. Coordenação motora de escolares do ensino fundamental: influência de um programa de intervenção. Journal of Physical Education, Maringá, v. 28, n. 1, p. e-2842, 2017.

FERRARI, G. L. Changes in adiposity levels in schoolchildren according to nutritional status: analysis over a 30 year period. Brazilian Journal of Kineanthropometry and Human Performance, Florianopolis, v. 15, n. 4, p. 405, 2013.

GOODWAY, J.; HAUBENSTRUCKER, J. Parent-assisted instruction in a motor skill program for at risk preschool children. Adapted Physical Activity Quarterly, Champaign, v. 16, p. 415-26, 1999.

HALLAL, P. C. Global physical activity levels: surveillance progress, pitfalls, and prospects. Lancet, London, v. 380, n. 9838, p. 247-57, 2012.

IIVONEN, S.; SÄÄKSLAHTI, A. K. Preschool children's fundamental motor skills: a review of significant determinants. Early Child Development and Care, Cidade, v. 184, p. 1107-26, 2014.

KIPHARD, E. J.; SCHILLING, V. F. Korper-koordination-test. Fur kinder KTK: Manual Von Fridhelm Schilling. Weinhein: Beltz Test; 1974.

LAGE, G. M. O efeito da interferência contextual na aprendizagem motora: contribuições científicas após três décadas de publicação do primeiro artigo. Revista Brasileira de Cineantropometria e Movimento Humano, Florianópolis, v. 19, n. 2, p. 107-19, 2011.

LAI, K. S. Do school-based interventions focusing on physical activity, fitness, or fundamental movement skill competency produce a sustained impact in these outcomes in children and adolescents? A systematic review of follow-up studies. Sports Medicine, Auckland, v. 44, p. 67-79, 2014.

LARKIN, D.; HOARE, D.; SMITH, K. Understanding and teaching children with movement dysfunction. Nedlands, Western Australia: Department of Human Movement and Recreation Studies, 1989.

LAZZARONI, P. A influência do clima motivacional para maestria nas habilidades motoras fundamentais: ênfase na patinação artística. Porto Alegre, 2017.

LEE, I. M. Effect of physical inactivity on major non-communicable diseases worldwide: an analysis of burden of disease and life expectancy. Lancet London, v. 380, n. 9838, p. 219-29, 2012.

LOPES, V. P.; MAIA, J. A. R. Efeitos do ensino no desenvolvimento da capacidade de coordenação corporal em crianças de 8 anos de idade. Revista Paulista de Educação Física, São Paulo, v. 11, n. 1, p. 40-8, 1997.

LOPES, V. P.; MAIA, J. A. R.; SILVA, R. G.; SEABRA, A.; MORAIS, F. P. Estudo do nível de desenvolvimento da coordenação motora da população escolar (6 a 10 anos de idade) da região autónoma dos Acores. Revista Portuguesa de Ciências do Desporto, Porto, v. 3, n. 1, p. 47-60, 2003.

MALINA, R. M.; BOUCHARD, C.; BAR-OR, O. Growth, maturation and physical activity. 2. ed. Champaign: Human Kinetics Books, 2009.

MAROCO, J. Análise estatística, com utilização do SPSS. 2.ed. Lisboa: Edições Sílabo, 2003. 
MEMMERT, D.; ROTH, K. Teaching invasion games: The effects of specific and non-specific concepts on creativity. Journal of Sport Science, London, v. 25 n. 12 , p. 1423-32, 2006.

MOREIRA, J. P.; LOPES, M. C.; MIRANDA-JÚNIOR, M. V.; VALENTINI, N. C.; LAGE, G. M.; ALBUQUERQUE, M. R. Körperkoordinationstest Für Kinder (KTK) for brazilian children and adolescents: factor analysis, invariance and factor score. Front Psychology, Lausanne, v. 10, n. 2.524, p. 1-11, 2019.

MORERA-CASTRO, M.; ARGUEDAS-VIQUEZ, R.; BRABENEC-AGUILAR, S. Efeito de uma intervenção motora baseada no método da descoberta guiada sobre os padrões básicos de movimento de uma criança de 9 anos: estudo de caso. MHSalud, Heredia, v. 17, n. 1, p. 34-48, 2020.

MOSSTON, M.; ASHWORTH, S. Ensino de educação física. Barcelona, Madrid: Hispano Europea, 1986.

NEWELL, K. M. On task and theory specificity. Journal of Motor Behavior Washington, v. 21, n. 1, p. 92-6, 1989.

PIFFERO, C. M.; VALENTINI, N. C. Habilidades especializadas do tênis um estudo de intervenção na iniciação esportiva com crianças escolares. Revista Brasileira de Educação Física e Esporte, São Paulo, v. 24, n. 2, p. 149-63, 2010.

PIOLA, T. S.; BACIL, E. L. D.; WATANABE, P. I.; COSTA, T. R. A.; CAMPOS, W. Correlatos da atividade física em crianças e adolescentes: um estudo piloto. Caderno de Educação Física e Esporte, Marechal Cândido Rondon, v. 16, n. 1 , p. 217-25, 2018

PLESS, M.; CARLSSON, M. Effects of motor skill intervention on developmental coordination disorder: a meta-analysis. Adapted Physical Activity Quarterly, York, v. 17, n. 4, p. 381-401, 2000.

POETA, L. S.; ROSA NETO, F. Intervenção motora em uma criança com transtorno de déficit de atenção/hiperatividade (TDAH). Lecturas Educación Física y Deportes, Revista Digital, Buenos Aires, v. 10, n. 89, 2005. Disponível em: <www.efdeportes.com/efd89/tdah.htm>. Acessado em: 3 de março de 2021.

REIS, R. S.; HINO A. A.; FLORINDO A. A.; AÑEZ, C. R.; DOMINGUES, M. R. Association between physical activity in parks and perceived environment: a study with adolescents. Journal of Physical Activity and Health, Champaign, v. 6, n. 4, p. 503-9, 2009.

ROTH, K.; KRÖGER, C. Escola da bola - um abc para iniciantes nos jogos esportivos. 2. ed. São Paulo: Phorte; 2006.

SAMPAIO, D.; VALENTI, N. C. Iniciação esportiva em ginástica rítmica: abordagem tradicional e o clima motivacional para a maestria. Revista da Educação Física, Maringá, v. 26, n. 1, p. 1-10, 2015.

SÁNCHEZ-LÓPEZ BAÑUELOS, F. Bases para uma didática da educação física e do esporte. Madrid: Gymnos, 1989.

SMITS-ENGELSMAN, B. C.; BLANK, R.; VAN DER KAAY, A. C.; MOSTERD-VAN DER MEIJS, R.; VLUGT-VAN DEN BRAND, E.; ... ; WILSON, P. H. Efficacy of interventions to improve motor performance in children with developmental coordination disorder: a combined systematic review and meta-analysis. Developmental Medicine \& Child Neurology, York, v. 55, p. 229-37, 2013.

SOUZA, M.; BERLEZE, A.; VALENTINI, N. C. Efeitos de um programa de educação pelo esporte no domínio das habilidades motoras fundamentais e especializadas: ênfase na dança. Revista da Educação Físca, Maringá, v. 19, n. 4, p. 509-19, 2008

STODDEN, D.; GOODWAY, J.; LANGENDEROFER, S.; ROBERTON, M. A.; RUDISILL, M.; GARCIA, C. A developmental perspective on the role of motor skill competence in physical activity: an emergent relationship quest. Quest, Cambridge, v. 60, p. 290-306, 2008.

TANI, G.; MANOEL, E.; KOKUBUN, E.; PROENÇA, J. Educação física escolar: fundamentos de uma abordagem desenvolvimentista. São Paulo, EDUSP, 1988.

VALENTINI, N. C. A Influência de uma intervenção motora no desempenho motor e na percepção de competência de crianças com atrasos motores. Revista Paulista de Educação Física, São Paulo, v. 16, n. 1, p. 61-75, 2002.

VALENTINI, N. C.; RUDISILL, M. E. Goal orientation and mastery climate: a review of contemporary research and insights to intervention. Estudos de Psicologia, Campinas, v. 23, n. 2, p. 159-71, 2006

VIDAL, S. M.; BUSTAMANTE, A.; LOPES, V. P.; SEABRA, A.; SILVA, R. G.; MAIA, J. A. R. Construção de cartas centílicas da coordenação motora de crianças dos 6 aos 11 anos da Região Autónoma dos Açores, Portugal. Revista Portuguesa de Ciências do Desporto, Porto, v. 9, n. 1, p. 24-35, 2009.

ZANELLA, L. M.; SOUSA, M. S.; BANDEIRA, P. F. R.; NOBRE, G. C.; VALENTINI, N. C. Crianças com sobrepeso e obesidade: intervenção motora e suas influências no comportamento motor. Motricidade, Ribeira de Pena, v. 12, n. 1, p. 42-53, 2016.

\section{AGRADECIMENTOS}

Os autores agradecem a Unidade de Ensino Integral (UEI) pela gentileza hospitalidade, que permitiram a condução do presente estudo

\section{CONFLITO DE INTERESSE}

Os autores do estudo declaram não haver conflito de interesses.

\section{FINANCIAMENTO}

Este estudo não teve apoio financeiro.

\section{ORCID E E-MAIL DOS AUTORES}

Maria Clara Soares de Oliveira Vaz (Autor Correspondente) ORCID: 0000-0001-9942-322X.

E-mail:mariaclaraovaz@hotmail.com

Wagner de Campos

ORCID: 0000-0003-3979-1017.

E-mail: wagner@ufpr.br

Marcio José Kerkoski

ORCID: 0000-0002-8339-2593.

E-mail:marciok@utfpr.edu.br

Gilmar Afonso

ORCID: 0000-0001-8761-0191.

E-mail: gafonso@utfpr.edu.br 